\title{
GEOMETRIC CONSTRUCTION OF CERTAIN HIGHEST WEIGHT MODULES
}

\author{
ROGER ZIERAU
}

\begin{abstract}
We identify certain singular unitary representations of semisimple Lie groups which were constructed by Rawnsley, Schmid and Wolf. The method is to identify them with the appropriate derived functor modules. Included are ladder representations of the indefinite unitary groups.
\end{abstract}

1. In [RSW] a method was given to unitarize certain highest weight modules of a reductive Lie group. The representations are realized on the Dolbeault cohomology groups of an indefinite Kähler semisimple symmetric space with coefficents in a holomorphic homogeneous vector bundle. They are unitarized by showing that the $G$-invariant indefinite inner product on the space of $L_{2}$ harmonic forms is positive semidefinite, and that its nullspace is the kernel of the natural map onto Dolbeault cohomology.

The purpose of this note is to show how to identify those representations which are unitarized by this method when the "highest $K$-type condition" of [RSW] fails. In particular, for the indefinite unitary groups $\mathrm{U}(p, q)$, all the ladder representations except one $\left(\nu_{p-q}^{*}\right.$, see $\left.\S 4\right)$ are included.

The result was obtained by Rawnsley, Schmid and Wolf by a case by case study, see [RSW, §11.8-§11.12]. However, it was not applied to the ladder representations of $\mathrm{U}(p, q)$ in cases where the highest $K$-type condition fails, see [RSW, §13]. The method here does not use a case by case analysis.

It should be mentioned that the unitary highest weight modules have been classified [EHW] as algebraically constructed quotients of generalized Verma modules. That algebraic construction is quite different from the geometric method considered here.

2. Let $G, H$ and $M$ be as in $\S 2$ of [RSW]. Thus $G / H$ is an indefinite Kähler semisimple symmetric space, $H=G^{\tau}=$ the fixed points of an involution $\tau$ and $M=G^{\theta \tau}$ where $\theta$ is a Cartan involution commuting with $\tau$. The Lie algebra of $G$ decomposes under $\tau$ as

$$
\mathfrak{g}_{0}=\mathfrak{h}_{0}+\mathfrak{q}_{0}, \quad \mathfrak{a}=\left(\mathfrak{a}_{0}\right)_{\mathbf{C}}=\mathfrak{a}_{+}+\mathfrak{a}_{-},
$$

where $a_{ \pm}$represent the holomorphic and antiholomorphic tangent spaces. Similarly, under $\theta$,

$$
\mathfrak{g}_{0}=\mathfrak{f}_{0}+\mathfrak{p}_{0} .
$$

Received by the editors September 19, 1984.

1980 Mathematics Subject Classification. Primary 22E46; Secondary 22E70.

Key words and phrases. Semisimple Lie group, unitary representation, Dolbeault cohomology, indefinite Kähler symmetric space, derived functor modules, ladder representation. 
$M / H \cap K$ and $K / H \cap K$ are hermitian symmetric spaces of the noncompact and compact type, respectively. Choose a $\theta$-stable fundamental Cartan subalgebra of $\mathfrak{g}$ contained in $\mathfrak{h}$ and fix a positive system of roots as in [RSW], $\mathfrak{a}_{+}$is a sum of positive root spaces.

There is a $C^{\infty}$ fibration $\pi: G / H \rightarrow K / H \cap K$ with typical fiber $M / H \cap K . \pi$ need not be holomorphic (see 2.21 in [RSW]).

Let $W$ be a unitary representation of $H$ with highest weight $\chi$. $G$ acts on the Dolbeault cohomology groups $V=H^{s}(G / H, \mathrm{~W})$ where $s=\operatorname{dim}_{\mathrm{C}}(K / H \cap K)$ and $\mathrm{W} \rightarrow G / H$ is the associated holomorphic homogeneous vector bundle. Consider the conditions (of [RSW]) on $\chi$ :

$L_{2}$ condition: $\langle\chi+\rho(\mathfrak{m}), \gamma\rangle<0, \forall \gamma \in \Delta\left(\mathfrak{p} \cap \mathfrak{q}_{+}\right)$.

Highest K-type condition: $\langle\chi+\rho(\mathfrak{f}), \alpha\rangle<0, \forall \alpha \in \Delta\left(\mathfrak{f} \cap \mathfrak{a}_{+}\right)$.

THEOREM [RSW]. Suppose $G / H \rightarrow K / H \cap K$ is holomorphic.

(a) $H^{p}\left(G / H, \mathbf{F}_{\chi}\right)_{K} \simeq H^{p}\left(K / H \cap K, \mathbf{H}^{0}\left(M / H \cap K, \mathbf{F}_{\chi}\right)\right), F_{\chi}$ the finite-dimensional $H$-module with highest weight $\chi$.

(b) If the highest $K$-type condition holds for $\chi$ then $H^{p}\left(G / H, \mathbf{F}_{\chi}\right)=0$, for $p \neq s$ and $V$ is the irreducible G-module with highest weight $w_{0}(\chi+\rho(\mathfrak{f}))-\rho(\mathfrak{f})$ (where $w_{0}$ is the element of the Weyl group of $K$ with $\left.w_{0}\left(\Delta^{+}(\mathfrak{h} \cap \mathfrak{f})\right) \subset \Delta^{+}, w_{0}\left(\Delta\left(\mathfrak{f} \cap \mathfrak{q}_{+}\right)\right) \subset-\Delta^{+}\right)$.

(c) If $\chi$ satisfies the $L_{2}$ condition then $V$ is unitary.

3. We assume that the $L_{2}$ condition holds and that the highest $K$-type condition may fail. Writing $H^{0}\left(M / H \cap K, \mathbf{F}_{\chi}\right)_{H \cap K} \simeq \bigoplus_{\tilde{n}} F_{-\tilde{n} \tilde{\gamma}} \otimes F_{\chi}$, where $\tilde{n} \tilde{\gamma}$ is as in 4.2 of [RSW], we get

$$
V_{k} \simeq \bigoplus_{\tilde{n}} H^{s}\left(K / H \cap K, \mathbf{F}_{-\tilde{n} \tilde{\gamma}} \otimes \mathbf{F}_{\chi}\right)
$$

When the highest $K$-type condition holds it is easy to see that $V_{K}$ has highest weight $w_{0}(\chi+\rho(\mathfrak{f}))-\rho(\mathfrak{f})$. This is because the highest possible $K$-type (when $\tilde{n}=0$ ) is $H^{s}\left(K / H \cap K, \mathbf{F}_{\chi}\right)$ and this is nonzero.

When the highest $K$-type condition fails this "expected" $K$-type does not occur $\left(H^{s}\left(K / H \cap K, \mathbf{F}_{\chi}\right)=0\right)$. If we could easily decompose $F_{-\tilde{n} \tilde{y}} \otimes F_{\chi}$ then we could write down all the $K$-types, using the Borel-Weil theorem. To avoid decomposing this tensor product we find the derived functor module with the same $K$-spectrum as $V$, then induce in stages (so we may start with a one-dimensional representation) and apply the "generalized Blattner formula" on p. 357 of [V1].

For details on derived functor modules see Chapter 6 of [V1]. We give some notation here. Let $l+\mathfrak{u}$ be a $\theta$-stable parabolic subalgebra of $g$. Let $\Gamma$ be the functor from ( $\mathfrak{g}, L \cap K)$-modules to $(\mathrm{g}, K)$-modules defined by taking the $K$-finite vectors, and let $\Gamma^{p}$ be the $p$ th right derived functor of $\Gamma$. Set $\left(L_{l+u}^{g}\right)^{p}(Y)=\Gamma^{p}\left(\mathrm{U}(g) \otimes_{l+u} Y\right)$ for any $(l, L \cap K)$-module $Y$.

Proposition. $V_{K} \simeq\left(L_{\mathfrak{h}+\mathfrak{a}_{+}}^{\mathfrak{g}}\right)^{s}\left(F_{\chi}\right)$, as $K$-modules. 
Proof. Let $\delta$ be a $K$-type, identify $\delta$ with its highest weight, and let $E_{\delta}$ be the space of $\delta$. The generalized Blattner formula on p. 357 of [V1] is given in terms of $R_{l+\mathrm{u}}^{p}=\Gamma^{p}\left(\operatorname{Hom}_{l+\mathfrak{u}}(\mathrm{U}(\mathrm{g}),-)_{L \cap K}\right)$. However, by $\S 5$ of $[\mathrm{V} 2], \quad\left(L_{l+\mathfrak{u}}^{\mathrm{g}}\right)^{p}(Y) \simeq$ $\left(R_{l+\bar{u}}^{\mathfrak{g}}\right)^{2 s-p}(Y)$ where $l+\overline{\mathrm{u}}$ is the opposite parabolic. Thus

$$
\begin{aligned}
\operatorname{dim} \operatorname{Hom}_{K} & \left(E_{\delta}, L^{s}\left(F_{\chi}\right)\right) \\
& =\sum_{j=0}^{s}(-1)^{j} \operatorname{dim} \operatorname{Hom}_{I \cap \mathfrak{f}}\left(H^{j}\left(\overline{\mathfrak{u}} \cap \mathfrak{f}, E_{\delta}\right), S(\overline{\mathfrak{u}} \cap \mathfrak{p}) \otimes F_{\chi+2 \rho(\mathfrak{u} \cap \mathfrak{f})}\right) \\
& =\sum_{\tilde{n}} \sum_{w \in W_{\mathcal{K}}^{\prime}}(-1)^{l(w)} \operatorname{dim} \operatorname{Hom}_{I \cap \mathfrak{f}}\left(F_{w w_{1}(\delta+\rho(\mathfrak{f}))+\rho(\mathfrak{f})}, F_{-\tilde{n} \tilde{\gamma}} \otimes F_{\chi+2 \rho(\mathfrak{u} \cap \mathfrak{f})}\right)
\end{aligned}
$$

where $W_{K}^{\prime}=\left\{w \in W_{K}\right.$ : if $\lambda$ is $\Delta^{+}(\mathfrak{f})$-dominant then $w \lambda$ is $\Delta^{+}(l \cap \mathfrak{f})$-dominant $\}$, $w_{1}=$ longest element of $W_{K}$.

Therefore, $L^{p}\left(F_{\chi}\right)_{K} \simeq \sum_{\tilde{n}, \delta}(-1)^{l(w)} \delta$, summing over $\delta=\left(\sigma_{0} w w_{1}\right)^{-1}(\mu+\rho(\mathfrak{f}))-$ $\rho(\mathfrak{f})$ with $\mu$ occurring in $\bigoplus_{\tilde{n}}\left(F_{-\tilde{n} \tilde{\gamma}} \otimes F_{\chi}\right), \sigma_{0}=$ longest element of $W_{H \cap K}$.

On the other hand,

$$
V_{K} \simeq \bigoplus_{\tilde{n}} H^{s}\left(K / H \cap K, \mathbf{F}_{-\tilde{n} \tilde{\gamma}} \otimes \mathbf{F}\right) \simeq \bigoplus_{\tilde{n}, p}(-1)^{s-p} H^{p}\left(K / H \cap K, \mathbf{F}_{-\tilde{n} \tilde{\gamma}} \otimes \mathbf{F}_{\chi}\right),
$$

a $K$-type $\delta$ (identified with its highest weight) occurs iff $\delta=w(\mu+\rho(\mathfrak{f}))-\rho(\mathfrak{f})$ where $\mu$ occurs in $\bigoplus_{\tilde{n}}\left(F_{-\tilde{n} \tilde{\gamma}} \otimes F_{\chi}\right)$. One can check that the signs and multiplicities are the same. Q.E.D.

Using the Borel-Weil theorem (see [EW]), we may write

$$
F_{\chi}=\left(L_{l+\mathfrak{h} \cap \mathfrak{u}}^{\mathfrak{h}}\right)^{s^{\prime}}\left(\mathbf{C}_{\chi^{\prime}}\right)
$$

for some parabolic $l+\mathfrak{u} \subset \mathfrak{h}+\mathfrak{q}_{+}, \chi^{\prime}$ orthogonal to $\Delta(l)$ and

$$
s^{\prime}=\frac{1}{2} \operatorname{dim}_{\mathbf{C}}(\mathfrak{h} \cap \mathfrak{f} / \mathfrak{h} \cap \mathfrak{f} \cap l) .
$$

By inducing in stages (6.3.10 of [V1]) we get

$$
V_{K} \simeq\left(L_{l+\mathfrak{u}}^{\mathfrak{g}}\right)^{s_{1}}\left(\mathbf{C}_{\chi^{\prime}}\right), \quad s_{1}=\frac{1}{2} \operatorname{dim}_{\mathbf{C}}(\mathfrak{f} / l \cap \mathfrak{f}) .
$$

Thus, we are inducing from a one-dimensional representation of $l$ and the generalized Blattner formula simplifies as follows:

Proposition.

$$
\begin{aligned}
& \operatorname{dim} \operatorname{Hom}_{K}\left(E_{\delta},\left(L_{l+u}^{\mathfrak{g}}\right)^{s_{1}}\left(\mathbf{C}_{\chi^{\prime}}\right)\right) \\
& =(-1)^{s_{1}} \sum_{w \in W_{K}}(-1)^{l(w)} p\left(\chi^{1}+\rho(\mathfrak{f})-w(\delta+\rho(\mathfrak{f}))\right)
\end{aligned}
$$

where $p(\gamma)$ is the number of ways to write $\gamma=\sum m_{\beta} \beta$ with $\beta \in \Delta(\mathfrak{u} \cap \mathfrak{p})$ and $m_{\beta} \geqslant 0$.

Proof. The generalized Blattner formula says the left-hand side is

$$
\begin{aligned}
& \sum_{j}(-1)^{j} \operatorname{dim} \operatorname{Hom}_{I \cap \mathfrak{f}}\left(H^{j}\left(\overline{\mathfrak{u}} \cap \mathfrak{f}, E_{\delta}\right), S(\overline{\mathfrak{u}} \cap \mathfrak{p}) \otimes \mathbf{C}_{\chi^{1}+2 \rho(\mathfrak{u} \cap \mathfrak{f})}\right) \\
& \quad=\sum_{w \in W_{K}^{\prime}}(-1)^{l(w)} \operatorname{dim} \operatorname{Hom}_{l \cap \mathfrak{f}}\left(\mathbf{C}, E_{w w_{1}(\delta+\rho(\mathfrak{f}))-\rho(\mathfrak{f})}^{*} \otimes S(\mathfrak{u} \cap \mathfrak{p}) \otimes \mathbf{C}_{\chi^{\prime}+2 \rho(\bar{u} \cap \mathfrak{f})}\right) .
\end{aligned}
$$


The latter summands give the number of times the trivial representation occurs in the tensor product. Now apply the formula on p. 142 of [H]. Q.E.D.

The next section shows how this proposition is used to identify the modules $V_{K}$.

4. Example. Let $G=\mathrm{U}(p, q)$, assume $p>q$. Let $H=\mathrm{U}(1) \times \mathrm{U}(p-1, q)$. The fibration $G / H \rightarrow K / H \cap K$ is holomorphic. Let

$$
\chi=-a \varepsilon_{1}\left(\varepsilon_{i}\left(\begin{array}{ccc}
t_{1} & & \\
& \ddots & \\
& & t_{p+q}
\end{array}\right] \equiv t_{i}\right) .
$$

The $L_{2}$ condition is $a \geqslant q+1$ and the highest $K$-type condition is $a \geqslant p$. If $a \geqslant p$ holds then $V$ is the ladder representation $\nu_{a-p}^{*}$ (in the notation of [SW] or [RSW], $\nu_{d}^{*}$ has highest weight $-d \varepsilon_{p}-\kappa$ if $d \geqslant 0$ and $-d \varepsilon_{p+1}-\kappa$ if $d<0$ where $\kappa \equiv \sum_{i=1}^{p} \varepsilon_{i}$ ). This calculation is done in $\S 13$ of [RSW].

Now consider the range $q+1 \leqslant a \leqslant p$. The unitarity is automatic but the representation must be identified. The possible $K$-types have highest weight

$$
\delta=-(a-p) \varepsilon_{p}-\kappa-\sum m_{i}\left(\varepsilon_{p}-\varepsilon_{p+i}\right) .
$$

The formula for the multiplicities of $K$-types given in $\$ 3$ shows that the first of these possible $K$-types which occurs is $-(a-p) \varepsilon_{p+1}-\kappa\left(m_{1}=p-a, m_{2}=m_{3}=\cdots\right.$ $=m_{q}=0$ ). This gives $\nu_{a-p}^{*}$ for $a=q+1, q+2, \ldots, p-1$.

By taking $H=\mathrm{U}(p, q-1) \times \mathrm{U}(1)$ the ladder representations obtained are $\nu_{d}^{*}$, $d \leqslant q-p-1$. In this case, since $p>q$, the $L_{2}$ condition implies that highest $K$-type condition.

In summary, the following ladder representations are unitarized $(p>q)$ :

\begin{tabular}{ccc}
\hline$\nu_{d}^{*}$ & $H$ & $\begin{array}{c}\text { highest } K \text {-type } \\
\text { condition }\end{array}$ \\
\hline$d \geqslant 0$ & $\mathrm{U}(1) \times \mathrm{U}(p-1, q)$ & holds \\
$0>d \geqslant q-p+1$ & $\mathrm{U}(1) \times \mathrm{U}(p-1, q)$ & fails \\
$q-p-1 \geqslant d$ & $\mathrm{U}(p, q-1) \times \mathrm{U}(1)$ & holds \\
\hline
\end{tabular}

The representation $v_{q-p}^{*}$ is realized on the Dolbeault cohomology groups using either parabolic but the $L_{2}$ condition fails for both parabolics, thus $\nu_{p-q}^{*}$ is not unitarized by these methods.

In this example $F_{\chi}$ is one-dimensional. When this is not the case one must find $\chi^{\prime}$ and the appropriate parabolic (see [A]). For example, take $H=\mathrm{U}(k) \times \mathrm{U}(p-k, q)$, $\chi=-\sum_{i=1}^{k} a_{i} \varepsilon_{i}, a_{1} \leqslant a_{2} \leqslant \cdots \leqslant a_{k}$. The Borel-Weil theorem gives $\chi^{\prime}=$ $-\sum_{i=1}^{k} a_{k+1-i} \varepsilon_{i}+\sum_{j=1}^{k}(k-(2 j-1)) \varepsilon_{j}$ and the parabolic $l+\mathfrak{u}$ has $l=u(1)^{k} \times$ $u(p-k, q)$. Now the formula of $\$ 3$ can be applied. 


\section{REFERENCES}

[A] J. Adams, Unitary highest weight modules, preprint. [EHW] T. Enright, R. Howe and N. Wallach, A classification of unitary highest weight modules, preprint. [EW] T. Enright and N. Wallach, Notes on homological algebra and representations of Lie algebras, Duke Math. J. 47 (1980), 1-15.

[H] J. Humphreys, Introduction to Lie algebras and representation theory, Springer-Verlag, 1972.

[RSW] J. Rawnsley, W. Schmid and J. Wolf, Singular unitary representations and indefinite harmonic theory, J. Funct. Anal. 51 (1983), 1-114.

[SW] S. Sternberg and J. Wolf, Hermitian Lie algebras and metaplectic representations, Trans. Amer. Math. Soc. 238 (1978), 1-43.

[V1] D. Vogan, Representations of real reductive Lie groups, Birkhäuser, 1981.

[V2] _ Unitarizability of certain series of representations, Ann. of Math. 120 (1984), 141-187.

Department of Mathematics, University of California, Berkeley, California 94720

Current address: Department of Mathematics, Massachusetts Institute of Technology, Cambridge, Massachusetts 02139 